\title{
SUJETOS, PREDICADOS Y NOMBRES PROPIOS
}

En este trabajo quisiera examinar algunos aspectos de la distinción sujetopredicado, en relación a la eliminación de los nombres propios en la notación lógica canónica, y algunas tesis relativistas. En la primera parte resumo parcialmente la explicación que da Strawson de tal distinción en Individuals ${ }^{1}$ y en la segunda considero la posición de Quine al respecto.

De acuerdo a F. P. Ramsey ${ }^{2}$ la distinción sujeto-predicado (S-P de aquí en adelante) es de naturaleza tal que para dudarla basta considerarla. La base de su escepticismo la explica Ramsey señalando que la tesis tradicional, de acuerdo a la cual si una proposición consiste de dos términos copulados, estos dos términos deben funcionar de diferente manera -uno como sujeto, el otro como predicado - no tiene ningún fundamento obvio. Por ejemplo, en la proposición "Sócrates es sabio", Sócrates es el sujeto, el ser sabio -o la sabiduría- el predicado. Sin embargo, nada nos impide invertir la proposición y decir: "La sabiduría es una característica de Sócrates", en este caso Sócrates, que era el sujeto, es ahora el predicado o, al menos, parte de él. Estas dos oraciones, "Sócrates es sabio". y "La sabiduría es una característica de Sócrates", insiste Ramsey, afirman el mismo hecho y expresan la misma proposición; el que sean distintas es tan importante a la lógica como el que dos oraciones, con el mismo significado pero en dos lenguas diferentes, sean distintas. Es cierto, sin embargo, que dado el par de oraciones mencionadas, muchas veces claramente preferiríamos usar una de ellas más que la otra, pero esto, nos dice Ramsey, es sólo una cuestión de estilo literario o del punto de vista desde el cual estemos abordando el hecho. Si estamos hablando acerca de Sócrates lo más natural es decir: Sócrates es sabio, mientras que si estamos discutiendo la sabiduría tendemos a decir: La sabiduría es una característica de Sócrates; lo importante es que, usemos la oración que usemos, estamos diciendo la misma cosa. Por lo tanto, cuál término sea el sujeto, cuál el predicado, depende de cuál oración usemos y esta última decisión no tiene nada que ver con la naturaleza lógica de Sócrates o la sabiduría, ya que es una cuestión puramente gramatical. Pero si esto es así entonces - concluye

1. Strawson, P. F., Individuals. Londres, Methuen, 1959.

2 Véase su articulo "Universals" recogido en The Foundations of Mathematics. Londres, Routledge \& Kegan Paul, 1931. 
Ramsey - no hay una distinción esencial entre el sujeto y el predicado de una oración ni tampoco podemos fundamentar otras distinciones ontológicas en ella.

Ahora bien, la verdadera importancia de este escepticismo radica, creo, más en la fuerza con la que plantea la necesidad de dar una explicación más completa de nuestra aceptación -o rechazo - de la distinción S-P que en los argumentos que de hecho da para rechazarla. Más que a negar la distinción, las observaciones de Ramsey invitan, parece, a hacer explícitas las razones que podamos tener para creer que la distinción S-P es una distinción lógicamente pertinente y no puramente gramatical. Por lo tanto, una respuesta más o menos completa a las dudas de Ramsey puede dividirse en dos partes. La primera consiste en tratar de mostrar que la distinción S-P no es sólo una distinción gramatical, sino que tiene como su razón de ser una distinción lógica. Para hacer esto debemos mostrar que sujetos y predicados son expresiones lógicamente asimétricas y debemos, al menos, incluir la enumeración y descripción de tales asimetrías. La segunda parte consiste en tratar de explicar esas asimetrias, esto es, en tratar de dar una explicación no formal de nuestras razones para distinguir, formalmente, entre sujetos y predicados; o sea, contrariamente a lo que sugiere Ramsey, la segunda parte de la respuesta se origina en la idea de que la distinción S-P debe, más que ser fundamento de otras distinciones ontológicas, ser ontológicamente fundamentada.

En Individuals, donde tal vez se encuentra la explicación más comprehensiva de la distinción S-P, encontramos dos criterios generales para distinguir entre sujetos y predicados. $^{3}$ El primero podría calificarse de gramatical, el segundo podría caracterizarse como categorial. Ambos, se arguye, reflejan asimetrfas que dan contenido a la doctrina tradicional de acuerdo a la cual los universales pueden aparecer ya sea como sujetos o ya sea como predicados, mientras que los particulares sólo pueden presentarse como sujetos.

Una primera aproximación a la formulación de un criterio es la siguiente: supongamos que en los asertos

\section{Sócrates es sabio Raleigh fuma}

queremos distinguir entre el sujeto y el predicado. Podriamos decir que los sujetos son aquellas expresiones que introducen y representan aquello de lo que estamos hablando, mientras que los predicados son las expresiones que introducen lo que queremos decir acerca de aquello que el sujeto representa.

3 Strawson ha investigado el problema de la distinción S-P en varios trabajos además de Individuals. (A partir de "Particular and General" (1953-1954) hasta su reciente Subject and Predicate in Logic and Grammar (1974)). Aquí limito sus puntos de vista a los de Individuals por razones de extensión y porque creo que alli podemos encontrar la estructura básica de su argumentación. Véanse, también, los cinco primeros ensayos de sus Logico. Linguistic Papers, Londres, Methuen, 1971 . 
Puesto que, parece, estamos hablando acerca de Sócrates y de Raleigh, podemos decir que "Sócrates" y "Raleigh" son las expresiones-sujeto de nuestros asertos y que "es sabio" y "fuma" son los predicados puesto que lo que queremos decir de Sócrates y Raleigh es que el primero es sabio y que el segundo fuma. Pero, obviamente, arguye Strawson asumiendo la posición escéptica, podria suceder lo contrario, podriamos estar hablando acerca de la sabiduria y del fumar, podríamos estar diciendo de ellos - la sabiduría y el fumarque son ejemplificados o practicados por Sócrates y Raleigh respectivamente. De la misma manera podríamos decir que "es sabio" y "fuma" representan a la sabiduría -o al ser sabio - y al fumar $\longrightarrow$ hábito de fumar- y anular así la efectividad del criterio. Esto no quiere decir que el criterio esté teóricamente infundado, ni tampoco que las nociones de 'un tema de discurso' y de 'representación' no tengan un papel que jugar en la formulación de un criterio, sino sólo que se requiere una base teórica para que el criterio, y las nociones correlativas, funcionen y no sean susceptibles a las críticas de Ramsey. El problema con el criterio bosquejado es que no toma en consideración la relación que existe, cuando se trata de determinar el asunto de una oración, entre dicha oración y el contexto en el que es usada.

Ahora, si adoptamos la terminología neutral de acuerdo a la cual "cualquier cosa que se introduce o puede introducirse en una observación a través de una expresión es un término", podremos decir con Strawson que el que las expresiones "Sócrates" y "es sabio" ("Raleigh" y "fuma") tengan en común el servir como medio para introducir un término no quiere decir que no haya una diferencia en la manera en que se lleva a cabo esta introducción. ¿Puede la gramática ayudarnos - podemos preguntar - a distinguir entre las diferentes maneras de introducir un término? Strawson sugiere que podemos distinguir entre la forma substantiva y la forma verbal de introducción y que esta distinción, aunque limitada y no explicada puede ser adecuadamente reforzada. Así, aún si expresiones como "es sabio" o "fuma" no se introducen siempre a través de un aserto -podríamos, por ejemplo, estar haciendo una pregunta - podemos decir que los predicados introducen sus términos en la forma asertiva o proposicional, puesto que las preguntas requieren respuestas y éstas claramente introducen proposiciones. Lo mismo se podría argüir en relación a los condicionales, cuando alguien dice: "Si Sócrates es sabio entonces..." dicha persona no está afirmando que Sócrates sea sabio, no se está comprometiendo a una opinión acerca del valor de verdad del antecedente del condicional, pero este antecedente, de hecho, introduce una proposición. Podemos así decir que la función primaria de la forma verbal de introducción de un término es la asertiva o proposicional y concluir que:

...el uso de la forma indicativa de un verbo involucra una forma característica de introducir el término que hace patente que aquello en 
lo que lo introducimos es una proposición. El uso de una forma substantiva, por otro lado, no tiene tales implicaciones; es aquella forma que naturalmente usariamos si lo que quisiéramos hacer fueran meras listas de términos. En la observación "Sócrates es sabio" ambas expresiones "Sócrates" y "es sabio" introducen términos; correspondientemente, Sócrates y ser sabio. Pero - recurriendo a una frase de W. E. Johnsonla expresión "es sabio". no sólo introduce el ser sabio sino que conlleva el vínculo asertivo o proposicional; o, en una terminología aún más vieja, no sólo introduce su término sino que también lo copula. (Individuals, p. 151.)

Éste no es todavía un criterio suficientemente estricto, no excluye el que descripciones definidas o expresiones substantivas tales como "todo", "algo" o "Sócrates es" sean clasificadas como expresiones predicativas. Para afinar el criterio Strawson agrega las siguientes restricciones: r) Una expresión sólo introduce un término si tiene, como parte de su uso normal, la función de distinguir ese término de otros; 2) Tanto el sujeto como el predicado deben ser expresiones capaces de dar lugar a una oración afirmativa al ser combinadas con una expresión adecuada de la clase contraria; 3) Un predicado es una expresión que no forma, por si misma, una oración completa.

Una vez que establecemos estas restricciones tenemos un criterio efectivo para distinguir "gramaticalmente" entre sujetos y predicados, lo hacemos distinguiendo entre frases substantivas y verbales y señalando que son los predicados y no los sujetos los que conllevan el "vínculo proposicional". Pero ¿es ésta una respuesta al escepticismo de Ramsey? Alguien podría argüir que la distinción entre frases substantivas y verbales es sólo una distinción gramatical, peculiar a las lenguas occidentales $y$, peor aún, no explicada puesto que, como Strawson mismo señala, "las clasificaciones gramaticales no declaran, unfvoca y claramente, su propia razón de ser lógica". Además, aún si el vínculo proposicional lo portan de hecho los predicados, este hecho no ha sido explicado o justificado e inclusive se podría poner en duda el que tal indicación proposicional deba ser localizada en el predicado; alguien podría sugerir que es independiente de la introducción de los términos.

Dada esta situación Strawson prosigue a dar una base más firme a la distinción por medio del desarrollo de un criterio categorial. El primer paso es pensar acerca de un término como un principio que recoge o reúne otros términos. Un término, podemos decir, reúne aquellos términos con los cuales puede establecer un vínculo asertivo cuyo resultado no es sólo una proposición significativa sino también verdadera. Estamos vinculando términos de esta manera cuando caracterizamos un objeto o cuando le atribuimos algo a alguna otra cosa o, también, cuando hablamos de un ejemplo o caso de algo. Las que siguen son algunas de las formas que usamos para establecer estos vínculos: “. . . se caracteriza por...”, “... es un ejemplo de...”, “...tiene la 
relación... con...". No debe suponerse, nos señala Strawson, que estas expresiones nombren relaciones puesto que los vínculos entre los términos en cuestión no dan lugar a relaciones ordinarias; apropiadamente los llama él "vínculos no-relacionales". Estos vínculos pueden enlazar particulares con particulares, universales con universales y particulares con universales.

Strawson distingue entre dos clases de universales: clasificantes y caracterizantes $\mathrm{y}$, consecuentemente, entre el vínculo clasificante o ejemplificante y el vínculo caracterizante. Un universal clasificante [sortal universal] se define como aquél que reúne particulares y provee un principio para distinguirlos y contarlos sin presuponer para ello un principio o método previo para individuar dichos particulares. Los universales caracterizantes se distinguen por el hecho de que, aun si poveen principios de agrupación, lo hacen sólo en relación a particulares previamente distinguidos o distinguibles por otros principios. Aproximadamente y con reservas - dice Strawson- algunos nombres comunes de particulares introducen universales clasificantes, mientras que verbos y adjetivos aplicables a particulares introducen universales caracterizantes.

Los particulares pueden también proveer principios para agrupar otros particulares pero, como en el caso de los universales caracterizantes, sólo lo hacen cuando tales particulares pueden ser previamente distinguidos por otro método ó principio previo. Así, de la misma forma que agrupamos aquellas acciones o aforismos que son sensatos, podemos agrupar aquellas acciones o aforismos que se le atribuyen a Sócrates; el particular Sócrates, por lo tanto, puede servir también como un principio de agrupación. Este vínculo norelacional entre particulares y particulares lo llama Strawson, en memoria de Cook Wilson, el "vínculo atributivo".

Veamos ahora cómo estos tres vínculos no-relacionales pueden sentar las bases para un criterio categorial. Empecemos por el vínculo clasificante. Notamos aquí que un mismo particular puede estar clasificatoriamente enlazado a diferentes universales clasificantes: Fido, un particular, ejemplifica los universales clasificantes "perro", "animal", "terrier", etc. En general, señalemos, los universales clasificantes pueden ser ordenados por un principio de subordinación. Por otro lado, un mismo universal clasificante puede estar relacionado a diferentes particulares por el vinculo clasificante: Fido, Coco, Rover, son particulares relacionados por el clasificante "perro". Los particulares asi relacionados, podemos decir, comparten una semejanza general o de clase. Vemos así que el principio por el cual los particulares agrupan o coleccionan universales es bastante distinto de aquel por el cual los universales agrupan o colectan particulares, aún si ambos lo hacen a través del vínculo clasificante. Strawson sugiere que esta diferencia la deberíamos de expresar dando a la forma " $x$ está relacionado ejemplarmente con $y$ " (" $x$ es un ejemplo de $y^{\prime \prime}$ ) no sólo una interpretación simétrica (en la que $x$ o y pueden ser 
ya sea particulares o universales, siempre y cuando uno pertenezca a una clase y el otro a la otra), sino también una asimétrica, una interpretación bajo la cual $x$ deba ser un particular y $y$ un universal.

De la misma forma, a través del vínculo caracterizante un mismo particular puede estar relacionado con varios universales caracterizantes: podemos caracterizar a Sócrates como sabio, conversador y mortal. Por otro lado cada uno de estos universales caracterizantes está relacionado por el mismo vínculo a muchos otros particulares: otras personas, además de Sócrates, pueden ser sabias, o conversadoras o mortales. Pero, de nuevo, aun si a través del vínculo caracterizante tanto particulares como universales pueden servir como principios para agrupar, esto no quiere decir que ellos reúnan sus co rrespondientes universales y particulares de la misma manera. Un particular reúne sus universales caracterizantes en base a su continuidad mientras que un universal caracterizante reúne a sus particulares en base a la "semejanza característica" de dichos particulares. Si esto es así deberíamos agregar, sugiere Strawson, la forma asimétrica " $x$ es caracterizado por $y$ " (donde $x$ debe ser un particular y $y$ un universal) a la forma simétrica " $x$ y $y$ están unidos por el vínculo caracterizante" ".

Puesto que, como vimos, un particular puede reunir por medio del vinculo caracterizante muchos universales caracterizantes, se sigue que debe también reunir, a través del vínculo atributivo, un cierto número de particulares. Esto es, si entre los universales caracterizantes reunidos por Sócrates están el ser sabio y el ser mortal, entonces, por el vínculo atributivo, Sócrates reúne ciertos actos particulares de sabiduría y una muerte particular, la suya. Por lo tanto podemos decir que mientras "sabio" y "mortal" pueden reunir, por el vinculo caracterizante, muchos particulares, una manifestación particular de la sabiduría de Sócrates o su muerte no puede, por el vínculo atributivo, reunir a ningún otro particular excepto a Sócrates. Strawson habla aqui del miembro independiente y el miembro dependiente en un vínculo atributivo: el primero es aquel que puede reunir otros particulares semejantes al miembro dependiente, mientras que éste se caracteriza por ser incapaz de reunir otros particulares. Así, a la forma simétrica, " $x$ está atributivamente vinculada a $y$ " podemos agregar la asimétrica " $y$ se atribuye a $x$ ", donde $y$ es el miembro dependiente.

En base a las simetrías y asimetrías mencionadas entre las formas en que los universales reúnen particulares y los particulares reúnen universales, podemos dar, argüye Strawson, una lectura básica o primaria a "y se predica de $x$ " que es: " $x$ se afirma como no-relacionalmente vinculada a $y$ ya sea como un ejemplo de $y$ o como caracterizada por $y$ ". Este sentido primario de predicación es una forma de la primera parte de la doctrina tradicional que dice que los universales pueden ser predicados de particulares pero no viceversa. La segunda parte - la que permite que los universales sean pre- 
dicados de universales- es fácilmente incorporada notando que los vínculos no-relacionales examinados pueden ser establecidos no sólo entre universales y particulares sino entre universales y universales. Strawson concluye que

...tomando como caso fundamental de: $y$ se predica de $x$, el caso en el que se afirma que $x$ (un particular) es un ejemplo de, o está caracterizado por y (un universal) y procediendo, a partir de allí, a desarrollar otros casos por analogía o extensión, podemos construir un sentido de 'predicar' de acuerdo al cual es verdad que los universales pueden tanto simplemente predicarse como tener cosas predicadas de ellos (esto es, ser sujetos), mientras que los particulares nunca se pueden simplemente predicar, aunque puedan tener cosas predicadas de ellos (esto es, ser sujetos) y pueden ser parte de lo que se predica. (Individuals, p. 172.)

Una vez establecido el criterio categorial que es independiente del "gramatical" Strawson procede a examinar las "tensiones y afinidades" entre estos dos criterios a fin de dar una explicación completa de la doctrina tradicional. Consideremos una de estas tensiones y su correspondiente afinidad.

El criterio categorial nos permite dar una respuesta más enérgica al escepticismo de Ramsey puesto que si lo aplicamos tanto a

a) Sócrates es sabio como a

b) La sabiduría es una característica de Sócrates

obtenemos el mismo resultado: la sabiduría se predica de Sócrates, ya que en ambas oraciones el particular, Sócrates, y el universal, sabiduría, están relacionados por el vínculo caracterizante. El criterio gramatical, por otro lado, no es tan definitivo en su veredicto cuando lo aplicamos a $b$ ), a menos que la reescribiéramos así

c) La sabiduria es socrática (socratiza).

En este caso, de acuerdo al criterio gramatical, deberíamos tal vez decir que Sócrates se predica de la sabiduría y, consecuentemente, los dos criterios resultarían incompatibles. Normalmente evadimos esta dificultad recurriendo al universal aparente "ser una característica de". Usándolo nos evitamos decir que Sócrates se predica de la sabiduría, esto es, evitamos c) puesto que hacemos que parezca que lo que realmente estamos predicando de la sabiduría no es un particular, Sócrates, sino un universal complejo que incluye a dicho particular. Pero, ¿es ésta una maniobra legítima? Supongamos que la queremos aplicar a $a$ ) y obtenemos

d) Sócrates se caracteriza por la sabiduría

Esta transformación, nos señala Strawson, sugeriría que estamos incorporando el vínculo no-relacional —el vínculo que en este caso une a Sócrates y la sabiduría - a uno de los términos. Pero, si una proposición sujeto-predicado es la afirmación de un vínculo no-relacional entre términos, entonces 
resultaria que la proposición estaría afirmando un vínculo no-relacional entre los nuevos términos, en este caso los introducidos por "Sócrates" y "caracterizarse por la sabiduria". La dificultad, arguye Strawson, es que una vez iniciado el proceso no hay razón para no aplicárselo también a la proposición resultante y obtener

e) "Sócrates se caracteriza por caracterizarse por la sabidurfa" y asi sucesivamente; si este fuera el caso nunca podríamos formular una proposición.

Esta objeción, sin embargo, sólo se aplicaría a b) -como alternativa de c) - si no tuviéramos ninguna razón para preferir la una a la otra, pero el criterio categorial nos provee con tales razones. Otra alternativa, que no requiere acudir al criterio categorial, sería considerar a $b$ ) como una perífrasis de a) y no como una forma de evadir c), o sea construir "La sabiduría es una característica de" como predicado y "Socrates" como sujeto pero entonces estaríamos negando la posibilidad -que originalmente el criterio gramatical dejaba abierta - de un análisis de $b$ ) en el cual la sabiduría apareciera como sujeto. Parece así que, dada la dificultad que presenta $b$ ), el criterio gramatical tiene ya sea que acudir a un universal aparente - "ser una característica de" - o ser reforzado con el criterio categorial. En cualquier caso podemos decir que ambos criterios no sólo no se contradicen sino que se complementan. Por otro lado, estos criterios y su afinidad de apli cación reciben su cabal explicación teórica a través de una distinción ulté rior entre las maneras en las que los términos se introducen en las proposiciones.

Una expresión que introduce un término debe, a fin de cumplir su función introductora, identificar o distinguir tal término. Por ejemplo, cuando decimos "Raleigh fuma", "Raleigh" identifica al particular al que nos estamos refiriendo, "fuma", la propiedad o característica que se le atribuye. Las condiciones requeridas por la introducción de un particular en una proposición las decribe Strawson en términos de referencias identificantes y en relación a un interlocutor y un oyente. Asi, las condiciones para hacer una referencia identificante a un particular son las siguientes: $i$ ) Debe haber un particular al que el interlocutor se esté refiriendo; $i i)$ Debe haber un particular que el oyente asuma ser aquel al que el interlocutor se está refiriendo; iii) El particular del interlocutor debe ser idéntico con el del oyente. Cómo, de hecho, se cumplen estas condiciones es una cuestión sumamente compleja de la cual aquí sólo daremos los lineamientos generales.

Para que una referencia identificante a un particular se lleve a cabo, nos dice Strawson, es necesario que exista una proposición -que el interlocutor en alguna forma conoce- que afirma que hay un particular que responde en forma única a cierta descripción. Esta condición también se aplica al oyente pero ello no quiere decir que la descripción a la que él acude deba 
ser la misma que usaría el interlocutor. En la introducción de un término universal, por otro lado, no encontramos condiciones paralelas, notoriamente, introducir un universal no involucra que conozcamos ninguna proposición empírica. La razón es la siguiente: supongamos que queremos determinar qué proposición empírica - si la hay- es necesaria para la introducción de un universal $\phi$. Saber que algo es $\phi$, podríamos decir, es condición suficiente para introducir $\phi$, pero -en el caso de que no se supiera de ningún ejemplo o caso de $\phi$ - también lo es el que nada sea $\phi$. Ahora, puesto que no hay una tercera posibilidad con respecto a la ejemplificación de $\phi$, podemos decir que la disyunción -o algo es $\phi$ o nada es $\phi$ - podría funcionar como una condición necesaria para la introducción de $\phi$, pero esta disyunción, notemos, no es una proposición empírica sino una tautología, una expresión que no afirma nada acerca del mundo.

El que la introducción de un universal $\phi$ no conlleva que conozcamos ninguna proposición empírica en particular se podría poner en duda arguyendo que proposiciones de la forma "algo es $\phi$ " no tendrían el significado que de hecho tienen a menos que un buen número de ellas fueran verdaderas; consecuentemente, se diría, no hay una distinción clara entre lo que se presupone al introducir un particular y lo que se presupone al introducir un universal. La situación, se podría argüir, se describe mejor diciendo que la introducción de un término particular presupone universalmente la verdad de alguna proposición empírica, mientras que la introducción de un término universal la presupone en general. A esta objeción Strawson da dos respuestas. La primera incluye el distinguir entre la clase de hechos afirmados por medio de las proposiciones que la introducción de particulares conlleva y aquellos afirmados mediante las proposiciones que la introducción de universales trae consigo. La introducción de un particular requiere de una proposición que afirme un hecho acerca del mundo, un hecho que pudiéramos decir pertenece a la historia, mientras que la proposición que necesitaríamos para introducir un universal no es de una clase determinada. El que algo, en algún lugar, en algún momento - nos dice Strawson- es o fue rojo, o redondo, o sabio, no es un hecho que pudiéramos incorporar a la historia. La segunda respuesta consiste en señalar que la introducción de un particular involucra no sólo una proposición empírica verdadera sino el que la sepamos verdadera. Por otro lado, para que una expresión introduzca un universal no es necesario, arguye Strawson, que quien la usa sepa o crea que dicho universal está de hecho ejemplificado. Podemos por lo tanto concluir que:

La introducción identificante ya sea de un particular o un universal en un discurso implica saber qué particular o qué universal se presenta, o se intenta introducir, a través de la expresión introductora. El saber qué particular se presenta implica conocer o, a veces -en el caso del oyente - enterarse, a través de la expresión introductora usada, de 
un hecho empírico suficiente para identificar ese particular y distinto al hecho de que él es el particular que, en ese momento, se está introduciendo. Sin embargo, saber qué universal se presenta no implica, de la misma forma, conocer un hecho empirico: implica meramente conocer el lenguaje. (Individuals, pp. 185-186.)

Las observaciones anteriores acerca de los presupuestos necesarios a la introducción de particulares y universales sugiere un tercer criterio para distinguir entre sujetos y predicados. Una expresión que introduce un particular conlleva, por el hecho de presuponer una proposición empírica, cierto "peso de los hechos" del que carece una expresión que introduce un universal. Las expresiones que introducen un universal sólo pueden "ayudar" a presentar un hecho y esto únicamente cuando forman parte de una afirmación explícita. Esta asimetría recuerda el criterio gramatical: las expresiones predicativas introducen sus términos en la forma asertiva o proposicional, esto es, en el estilo explícitamente incompleto que demanda ser consumado en un aserto. Tenemos aquí entonces un paralelo que es el punto de partida del siguiente criterio "mediador": una expresión-sujeto es aquella que, en un sentido, presenta un hecho por sí misma y es, en esa medida, completa; una expresión-predicado es aquella que en ningún sentido presenta, por sí misma, un hecho y es, en esa medida, incompleta. Este nuevo criterio está en conformidad tanto con el criterio gramatical como con el categorial; con este último porque las expresiones que introducen particulares nunca pueden ser incompletas y por lo tanto nunca pueden ser expresiones predicativas. Por otro lado, de acuerdo al criterio mediador ciertas expresiones que introducen universales son incompletas $y$, por tanto, pueden ser expresiones predicativas, pero otras, que también sirven para introducir universales -las que los identifican por descripción- son completas de acuerdo al nuevo criterio y, por lo tanto, pueden ser consideradas como sujetos. Por ejemplo, la sabiduría, puede ser introducida como "la cualidad más frecuentemente atribuida a Sócrates en ejemplos filosóficos". En este caso la introducción del universal involucra la verdad de una proposición empirica claramente destacable. Sin embargo alguien podría argüir que los universales no sólo pueden ser introducidos como sujetos por medio de descripciones definidas; a veces, se nos señalaría, los introducimos directamente, como cuando decimos: la sabiduría es una virtud. En este caso el criterio mediador falla, pero el remędio, nos sugiere Strawson, está al alcance de la mano: los vínculos no-relacionales que, vimos, unen particulares con universales y universales con universales. Estos vínculos, vimos también, sugerían que había un sentido básico en el que $y$ se predica de $x$, pero esto no excluye la extensión de la noción básica, siempre y cuando se preserven las analogías necesarias. Podemos decir así que ciertos universales se pueden predicar de otros porque podemos establecer entre ellos vínculos no-relacionales semejantes a los víncu- 
los caracterizantes y clasificantes que existen entre universales y particulares.

Un caso que puede ayudar a hacer más claro el criterio mediador es el siguiente: supongamos que alguien nos hace notar que ciertos predicados son expresiones complejas, predicados como "está casado con Maria" incluyen además del elemento universal un particular: María. ¿Quiere esto decir -podemos preguntar - que el predicado "estar casado con María" por incluir una expresión que introduce un particular es una expresión completa en el sentido examinado? De ser completa, esto es, si introdujera un hecho, la expresión "estar casado con María" no sería un predicado de acuerdo al criterio mediador. Que la expresión es incompleta, se hace evidente si consideramos que el único "hecho" — si insistimos en asf llamarle_ que introduce es el expresado por la tautología: "O hay alguien casado con María o no hay alguien casado con María."

Terminemos aquí esta primera parte haciendo hincapié en que los criterios examinados no substituyen a las tesis en torno a las cuales giran y en virtud de las cuales son formulados; por tanto encontrar dificultades en la aplicación o formulación de dichos criterios no implica necesariamente el cuestionar la verdad de las tesis en las cuales se originan. Por otro lado estos criterios y las ideas en torno a las cuales están construidos constituyen, creo, una respuesta excepcionalmente completa al escepticismo de Ramsey. Esto no quiere decir, sin embargo, que la distinción no pueda ser sometida a otras formas, más sutiles tal vez, de duda escéptica; las ideas de Quine, que procedemos ahora a considerar, son un ejemplo notorio de este escepticismo.

\section{II}

Las ideas de Quine que parecen estar en conflicto con la distinción S-P son aquellas relacionadas con la eliminación de los nombres propios de la notación canónica. La tesis en su forma más general es la siguiente: en un lenguaje adecuadamente reglamentado (lógicamente organizado) todos los términos singulares, con excepción de las variables de cuantificación, son asimilables - por un proceso de análisis_ a la categoría de términos generales. La posición de Quine, por tanto, es claramente antagónica, si no a la distinción S-P misma, sí a la importancia que se le da en el análisis de Strawson.4 Como vimos, Strawson no sólo quiere mostrar que, contrariamente a lo que pensaba Ramsey, es posible distinguir lógicamente entre sujetos y predicados, sino también que tal distinción es tan fundamental como la dis-

4 Esto no quiere decir que los análisis de Quine y Strawson no coincidan en muchos otros puntos que no consideramos aquí. Parte de las coincidencias, y diferencias, entre Quine y Strawson pueden verse en Individuals (especialmente las pp. 194-198), "Singular Terms and Predication", recogido en Logico-Linguistic Papers; Word and Object, parágrafo 38 (especialmente la nota en la p. 185) y D. Davidson y J. Hintikka (eds.), Words and Objections. Reidel, Dordrecht Holanda, 1969, pp. 320-325. 
tinción entre particulares y universales. Quine, por otro lado, piensa que, independientemente de que distingamos entre sujetos y predicados, tal distinción no puede tener la importancia que le da Strawson puesto que las expresiones que paradigmáticamente consideramos como sujetos son sistemáticamente eliminables y por lo tanto esencialmente superfluas. Dada esta diversidad de opiniones tratemos ahora de ver cuál de ellas se ajusta mejor a los hechos, no obstante que, en cierta forma, este criterio no puede tener mucha fuerza frente a una posición relativista.

El escepticismo de Quine consiste en argüir que aún si podemos, con cierto ingenio y aplicación, formular un criterio o conjunto de criterios a fin de distinguir entre sujetos y predicados, la distinción misma está limitada a nuestro lenguaje y es dudoso que pudiera generalizarse a todos los lenguajes. En este sentido podríamos tal vez decir que la distinción, si existe, no es lógica sino gramatical ya que el hecho de que sea provincial abre un hiato entre ella y una distinción lógica, universal. Esta posición tiene como telón de fondo teórico la tesis de la indeterminación de la traducción y en esta medida ha resultado ser inmune a aquellas críticas que, como las de Strawson, señalan que nuestra inteligencia de la notación canónica -una noción indudablemente lógica ${ }^{5}$ - depende de nuestra inteligencia de una distinción paralela a la de sujetos y predicados: la distinción entre términos singulares y términos generales en posición predicativa. Nuestro aparato de individuación, arguye Quine, está sujeto a la indeterminación de la traducción y, en esa medida, lo podemos considerar como provincial. Respondiendo a àlgunas de las criticas de Strawson nos dice Quine:

Argüí en Palabra y Objeto que la referencia objetiva está sujeta a la indeterminación de la traducción. Esta indeterminación afecta a la totalidad del aparato peculiarmente referencial de cuantificación, los pronombres, la identidad, la predicación y la distinción entre singular y general. Todo este aparato, y con él la cuestión ontológica misma, es provincial en este sentido: sólo es identificable en otras lenguas en relación a hipótesis analíticas de traducción que bien podrían haber tomado otros lineamientos. (Words and Objections, p. 320.)

Pero si la indeterminación de la traducción y la relatividad que ella conlleva se extienden hasta afectar a la totalidad de nuestro aparato de individuación, entonces, parecería, no podemos deshechar o cuestionar una distinción sólo porque es provincial pues en ese caso bien podríamos desechar o cuestionar nuestra noción de referencia objetiva, ya que, de acuerdo a Quine, también está sujeta a la indeterminación. Obviamente esto no quiere decir que aceptemos -o, para el caso, que rechacemos- los argumentos que con-

s Pero cuya universalidad, deberíamos agregar, no es siempre reconocida por Quine. 
ducen a la tesis de la indeterminación sino solamente que aún si esos argumentos fueran correctos eso no implicaría de por sí el que cuestionáramos la distinción S-P. Después de todo parece que las únicas nociones que escapan a la indeterminación son las de "sinonimia-estímulo" y "analiticidadestímulo" ya que ellas gozan, nos dice Quine, de la cualidad de ser aplicables a todos los lenguajes. Sin embargo, aun si podemos argüir que el ser provincial no es una buena razón para dudar o cuestionar la distinción S-P no quiere esto decir que ello baste para disipar el escepticismo de Quine. Dentro de los límites generales de la provincialidad que, según Quine, afecta a nuestro aparato de individuación, podemos separar aquellas nociones que son lo suficientemente importantes para ser reflejadas en nuestra notación canónica o que deberían ser entendidas a fin de entender dicha notación, de aquellas nociones que no son pertinentes a la notación canónica o a nuestra comprensión de ella. En este último grupo de nociones Quine coloca la función identificante de los términos singulares, la distinción S-P y la noción de un tema de discurso; incluidas en el primer grupo podrían tal vez estar las de la identidad y la predicación. En su respuesta a Strawson Quine señala que

...en un sentido Strawson está en lo correcto cuando dice que no explico la distinción entre general y singular sino sólo la forma de representarla. Estaria él equivocado si supusiera que yo crei que había o que deberia haber hecho algo más. (Words and Objections, p. 320 , el énfasis es mfo.)

Pero entonces podemos preguntar: ¿Cuál es la naturaleza del escepticismo de Quine? Por un lado hemos visto que la provincialidad no es por sí misma suficiente para poner en duda una noción, pero, por el otro, parece que Quine no cree que sea importante explicar la distinción entre general y singular, aun si admite que el esquema de predicación " $F x$ " y la distinción entre general y singular están intimamente ligados. En el fondo, nos dice Quine, la distinción entre general y singular es la distinción entre el papel de " $F$ " y el papel de " $x$ " en " $F x$ ", sin embargo esta relación sólo explica, admite Quine, una cosa en términos de la otra.

Una respuesta general que Quine posiblemente daría es que su escepticismo es el resultado de dos factores; el primero de ellos es que en la notación canónica podemos prescindir de los nombres propios, esto es, de la clase de expresiones que paradigmáticamente relacionamos con los sujetos. El segundo consiste en señalar que aún si nuestra comprensión de la notación canónica está relacionada o depende de nuestra comprensión de otras nociones, no hay una conexión necesaria entre la notación canónica y tales nociones puesto que ellas son, hemos visto, provinciales. Pero, si como Quine cree, la provincialidad afecta a la totalidad de nuestro aparato referencial entonces bien podemos limitar su escepticismo, en relación a la distinción S-P, a su idea de que 
los nombres propios son eliminables de la notación canónica. Pero, debemos preguntar, djustifica la eliminación de los términos singulares tal escepticismo? Quine, dijimos, cree que los nombres no son esenciales a la notación canónica porque cree que todo término singular, con excepción de las variables de cuantificación, es esencialmente prescindible. Dejando de lado la posible eliminación de las variables ${ }^{6}$ examinemos el análisis a través del cual son eliminados los nombres propios.

Tomemos la oración "Aristóteles era calvo"; usando la notación canónica podemos vertirla en

\section{$\mathrm{Ca}$}

Con la ayuda de variables, cuantificadores y un predicado ad hoc -aristoteliza- podemos remplazar, siguiendo el clásico análisis de Russell, (1) por

$$
\text { 业 } x(A x \&(y)(A y \supset \cdot x=y) \& C x)
$$

esto es, por un enunciado existencial que incluye una condición de unicidad. En (2) no tenemos nombres y los únicos términos singulares son las variables de cuantificación; por un proceso similar podemos eliminar las descripciones definidas.

En contra de la pulcritud lógica de este procedimiento alguien podría argüir que la reducción de (1) a una expresión puramente predicativa no es completa ya que la expresión (2) incluye un enunciado de identidad. ¿Cómo -podríamos preguntar - debemos entender ese enunciado? Supongamos que nuestra notación canónica ha sido modelada conforme a los lineamientos de Principia Mathematica, en ese caso podríamos apelar a la definición de identidad de Principia

$$
x=y .=:(\Phi) \Phi ! x \supset \Phi ! y \quad \text { Df. }
$$

y reescribir (2) asi:

$$
\operatorname{G} x(A x \&(y)(A y \supset(\Phi)(\Phi ! x \supset \Phi ! y)) \& C x)
$$

que sugiere algo así como una estratificación de predicados: el predicado - si predicado es- " $A$ " no es un predicado de primer orden."

Quine, obviamente, podría recordarnos que él no sólo no acepta la definición de Principia - y todo lo que ella conlleva - sino que nos ofrece una versión alternativa de la identidad que está libre de las implicaciones meta-

- Véase el ensayo "Variables explained away", recogido en el libro de Quine Selected Logical Papers, Nueva York, Random House, 1968.

7 Otra versión de la misma idea podría obtenerse si no restringiéramos el ámbito de $\phi$ a funciones predicativas pero entonces nos veriamos precisados a convertir en primitiva la noción de identidad. Véase Principia Mathematica (Introducción y ${ }^{* 13}$ ). 
físicas que aquejan a la definición de Russell. La versión que da Quine de la identidad es una función de los predicados disponibles en un lenguaje particular. Supongamos, nos invita Quine, un lenguaje que cuenta con un número finito de predicados clasificantes [sortals], monádicos y poliádicos, además de funciones de verdad y cuantificadores, en este caso:

Podemos definir ' $x=y$ ' por agotamiento del léxico de la siguiente manera. Formamos una conjunción de bicondicionales, con o sin cuantificadores universales. Un bicondicional ' $F x \equiv F y$ ' para cada clasificante monádico del léxico en el papel de ' $F$. Los bicondicionales cuantificados ' $(z)(F x z \equiv F y z)$ ' y ' $(z)(F z x \equiv F z y)$ ' para cada clasificante diádico. Correspondientemente para los clasificantes triádicos y poliádicos si los hay en el léxico. La conjunción de todos estos bicondicionales, con sus correspondientes cuantificadores, se puede tomar como la definición de $' x=y$ ' para el lenguaje extensional reglamentado que posee el léxico en cuestión. ${ }^{8}$

$\mathrm{Si}$, simplificando el caso, asumimos que el lenguaje en cuestión tiene, además de " $A$ ", sólo dos predicados monádicos " $B$ " y " $C$ " entonces podemos reescribir (2) así:

$$
\exists x(A x \&(y)(A y \supset \cdot B x \supset \cdot B x \equiv B y \& C x \equiv C y) \& C x)
$$

Hasta aquí todo parece bien; sin embargo debemos notar que la versión de la identidad que nos ofrece Quine conlleva ciertas dificultades ontológicas. Supongamos que aceptamos la mencionada definición, que aceptamos que la identidad es siempre relativa a un léxico, entonces debemos aceptar que los valores de nuestras variables - la finura de la individuación - varía de acuerdo a la riqueza de nuestro vocabulario; los objetos se constituirían como "mínimos lógicos", los productos finales de todas las distinciones disponibles.

Esta última idea es una forma de la llamada ontologia relativa, de la tesis de acuerdo a la cual la pregunta acerca de cuáles son los objetos de una teoria dada no tiene sentido excepto como una pregunta que correlaciona los objetos de esa teoría con los objetos, similarmente abiertos a consideración, de otra teoría. ${ }^{\ominus}$ Quine claramente acepta la idea de relacionar las dos tesis; identidad y ontología reflejan así las dos caras de una misma posición relativista. La identidad, nos dice Quine, forma una unidad con la ontología ya que no podemos saber qué sea una cosa sin saber cómo se diferencia de otras. Además la identidad también es relativa ya que, por un

8 Esta definición se encuentra en un artículo no publicado de Quine titulado "Identity", pero también puede verse en su Philosophy of Logic. Englewood, Prentice-Hall, 197o.

9 Véase el artículo "Ontological Relativity" recogido en Quine, W. V. O., Ontological Relativity and other Essays. Nueva York, Columbia University Press, 1969. 
lado, podemos definir una relación de identidad por agotamiento de un léxico $\mathrm{L} y$, por otro, el preguntar acerca de dicha identidad sólo tiene sentido con respecto a otra relación de identidad, definida en otro lenguaje en base a otro léxico. Pero entonces, como Quine mismo señala, la relativización de lá ontología da origen a un regresus que en algún punto debe parar. La dificultad es la siguiente: si las preguntas acerca de la referencia sólo tienen sentido en relación a un lenguaje dado, entonces, evidentemente, las preguntas concernientes a la referencia en tal lenguaje sólo tendrán sentido, a su vez, en relación a otro lenguaje ulterior. La situación es similar, nos dice Quine, a la que se presenta cuando preguntamos cuál es la posición de un objeto; normalmente la respuesta a este tipo de pregunta se da en relación a un sistema de coordenadas, con lo cual se da pie a que preguntemos cuál es la posición del sistema mismo - cuál es la posición del origen y la orientación de los ejes- y tengamos que recurrir a otro sistema de coordenadas para poder contestar la pregunta. El proceso, teóricamente, no tendría fin. En la práctica, sin embargo, siempre podemos recurrir a la ostensión y poner término al regresus generado. En el caso de la referencia el equivalente de la ostensión es nuestra lengua materna y la interpretación literal de sus palabras. ${ }^{10}$

Dejando por ahora de lado el problema de determinar el grado de relatividad de una posición que recurre en forma tan esencial a un lenguaje básico, notemos que hay una estrecha relación entre nuestras consideraciones ontológicas y dicho lenguaje básico [home language], el lenguaje en el cual formulamos la distinción S-P. Señalar esto, no obstante, no es suficiente, argüiría un seguidor de Quine, para dar a la distinción S-P la importancia que se le da, por ejmplo, en el análísis de Strawson. Su importancia, sin embargo, la podemos encontrar en su capacidad para poner en duda el relativismo de Quine.

Para ver así la distinción notemos primero que dicho relativismo puede tomar varias formas. Una de ellas es su versión de la identidad en términos del agotamiento de un léxico, otra es la tesis de la ontología relativa. El problema que la definición de Quine confronta en tanto que fuente de relatividad ontológica es su incompatibilidad con la lectura normal -irrestricta- de los cuantificadores de la notación canónica. Normalmente cuando leemos, por ejemplo, el cuantificador universal, no restringimos su ámbito a los objetos especificables de acuerdo a un léxico $L$, sino que solamente decimos: "Todo objeto $x$...". Por otro lado es verdad que podemos decir, con Quine, que identidad y ontologia van de la mano y que, en ese sentido, si la identidad es relativa a un léxico entonces la correspondiente ontologia lo debería ser también, pero esto no quiere decir que podamos modi. 
ficar - por alteración del léxico correspondiente- el dominio de nuestros cuantificadores; podemos definir un dominio definiendo una relación de identidad, de la manera en que lo hace Quine, y después cambiar ese dominio cambiando la relación de identidad, sin dar por ello lugar a ninguna posición relativista. La identidad no es una relación que pueda ser, como lo es la de similaridad, refinada, o graduada, sino que sólo la podemos cambiar puesto que alterándola cambiamos el dominio de nuestros cuantificadores. ${ }^{11}$

La otra tesis de Quine, la relacionada con la cuestión de determinar cuáles son los objetos de una teoría, se ve forzada, hemos visto, a recurrir a un lenguaje básico en el cual las palabras son tomadas en su valor literal. Hacer esto, me parece, conlleva el debilitamiento de su posición relativista y, consecuentemente, de su posición escéptica en relación a la distinción S-P. Las razones son las siguientes: en primer lugar, como vimos en la primera parte, el análisis que lleva a cabo Strawson de la distinción S-P sugiere que debemos distinguir entre los casos primarios y los casos secundarios de predicación, los primeros involucran un elemento no-lingüístico: la presuposición de un hecho empírico. Ahora bien, si los únicos objetos introducidos en nuestro discurso fueran objetos definidos en términos de una relación de equivalencia -en términos de un léxico de predicados dado- no podríamos objetar la relatividad de Quine, ya que la introducción de los predicados no involucra, hemos visto, ninguna proposición empírica, no requiere de otro conocimiento que no sea el del lenguaje. Sin embargo, si bien es cierto que, de acuerdo a Quine, las cuestiones ontológicas sólo tienen sentido en relación a un lenguaje dado, también lo es el que finalmente tenemos que recurrir a un elemento no-lingǘstico; el señalar, notemos, no es sólo conveniente para poner fin a un regresus de sistemas de coordenadas. Por otro lado, vimos, la introducción de particulares a través de nombres propios no es fácilmente reducible a la introducción de una conjunción de predicados, aun si reforzamos dicha conjunción con un cuantificador existencial y una condición de unicidad.

En segundo lugar, si no podemos aplicar a todos los casos el modelo de introducción de objetos por medio de relaciones de equivalencia (cuyo paradigma es la introducción de los números cuando definidos en términos de teoría de conjuntos), entonces no podemos generalizar la versión de Quine de la identidad en términos de un léxico. Aún más, dicha versión no puede ser generalizada ni siquiera al nivel de nuestro lenguaje básico ya que carecemos de una caracterización adecuada tanto de él como de su correspondien. te léxico. ${ }^{12}$

11 Esta observación la hace Michael Dummett en un artículo no publicado. Véase el capitulo 16 de su libro Frege, Londres, Duckworth, 1979.

12 Esta dificultad la he discutido en "Identidad y relatividad" que aparecerá en Actas del V Simposio de Lógica y Filosofía de la Ciencia, Universidad de Valencia, España, 1975. 
Si aceptamos la primera razón podemos argüir que el hecho de ignorarla, esto es, ignorar que hay una forma primaria de predicación y que ésta implica la presuposición de un hecho empírico, está a la base del relativismo de Quine. Únicamente por creer que todos los términos singulares pueden ser reducidos a expresiones predicativas — sin que por ello se pierda ningún elemento importante, no provincial - puede Quine aprobar la idea de que los objetos se pueden ver como "mínimos lógicos" los productos finales de todas las distinciones disponibles. Si este es el caso entonces podemos decir que la tarea de fundamentar distinciones "provinciales" tales como la distinción S-P, tienen, al menos, una importante función regulativa en nuestro aparato conceptual: marcar un límite a nuestras construcciones teóricas.

Instituto de InVEstigaciones Filosóficas.

RAúl QUESADA

Universidad Nacional Autónoma de México. 